\title{
Transplacental Transmission of SARS-CoV-2 Infection: A Case Report from Iran
}

\author{
Farhad Abolhasan Choobdar ${ }^{1}$, Maral Ghassemzadeh (iD ${ }^{2,}{ }^{*}$, Mohammad Attarian ${ }^{3}$, Ezzat Abbariki ${ }^{2}$, \\ Alireza Nateghian (iD ${ }^{1}$, Behrooz Ghanbari (iD) ${ }^{4}$, Samira Shah Hamzehi ${ }^{5}$, Melika Razavi Hashemi ${ }^{6}$ and \\ Zahra Azarbin $^{7}$ \\ ${ }^{1}$ Department of Pediatrics, Ali Asghar Children Hospital, Iran University of Medical Sciences, Tehran, Iran \\ ${ }^{2}$ Firuzgar General Hospital, Iran University of Medical Sciences, Tehran, Iran \\ ${ }^{3}$ Neonatology Department of Firuzgar General Hospital, Iran University of Medical Sciences, Tehran, Iran \\ ${ }^{4}$ Gastrointestinal and Liver Diseases Research Center, Firuzgar Hospital, Iran University of Medical Sciences, Tehran, Iran \\ ${ }^{5}$ Radiologist, Tehran, Iran \\ ${ }^{6}$ Department of Pathology, Gastrointestinal and Liver Diseases Research Center, Firuzgar Hospital, Iran University of Medical Sciences, Tehran, Iran \\ ${ }^{7}$ Department of Nursing, Firuzgar Hospital, Iran University of Medical Sciences, Tehran, Iran \\ "Corresponding author: Firuzgar General Hospital, Iran University of Medical Sciences, Postal Code : 1966676141, Tehran, Iran. Email: maral.ghassemzadeh1985@gmail.com
}

Received 2020 August 23; Revised 2020 September 29; Accepted 2020 November 05.

\begin{abstract}
We report a case of SARS-CoV-2 vertical transmission through the placenta in a neonate whose mother had non-M3 acute myeloid leukemia (AML) that was complicated with Covid-19 in the last trimester. Viral load in nasopharyngeal swabs from mother and neonate were high. Real-time PCR of the fetal side of the placenta was positive for SARS-CoV-2, which makes it possible to consider this case as a congenital case of SARS-CoV-2 infection that is transmitted through vertical transmission.
\end{abstract}

Keywords: Infection, Congenital, Premature, Immunosuppression

\section{Introduction}

SARS-CoV-2 infection is the first pandemic and the most challenging global concern of the current century. One of the most controversial debates is severe acute respiratory syndrome Coronavirus-2 (SARS-CoV-2) vertical transmission in pregnancy. Although its main transmission route is through droplets, other ways of transmission are just hypotheses (1-4). There are some case reports about its perinatal transmission, but it is not clear whether, in those cases, the transmission was vertical or through exposure to an infected environment (such as labor or operation room), infected caregivers, and infected secretions of mother or contact with family members (5). There is still a challenge about how the virus is transmitted during pregnancy from mother to the fetus (1). To have a clear definition for cases of probable perinatal SARS-CoV-2 transmission, a new type of classification is mentioned upon which congenital infection is definitely considered when the virus is found in the samples, which are obtained from unruptured and clean amniotic fluid or neonatal blood immediately after birth $(6,7)$. Collecting all of these samples, including placenta, amniotic fluid, cord blood, and pharyngeal swab, uninterruptedly after birth and in the first hours of life with sterile methods, reduces the risk of sample contamination and confirms congenital infection (8). In cases with positive placental findings, if amniotic fluid and neonatal blood have not been tested or have been reported negative in terms of SARS-CoV-2 Real-time polymerase chain reaction (RT-PCR), it should be classified only as "probable" congenital SARS-CoV-2 infection $(5,7)$. Up to now, there are not many papers that address the issue of Coronavirus disease 2019 (Covid-19) vertical transmission definitely. The results are not consistent even in these limited studies.

\section{Case Presentation}

A 30- year- old, gravid 1, para 0, abortion 0, woman was hospitalized in the obstetric ward of our center on May 13, 2020 . She was a primiparous woman $\left(23^{+6}\right.$ weeks of gestation). Her complaint was palpitation and lethargy, which was started a month before this visit, and she linked these symptoms to her pregnancy. Also, her routine laboratory check-up tests for pregnancy revealed pancytopenia: (WBC $=2.9 \times 1000 / \mathrm{mm}^{3}$, Lymphocyte $=35 \%, \mathrm{Hb}=6.6 \mathrm{~g} / \mathrm{dL}, \mathrm{RBC}=$ 2.1 Mill $/ \mathrm{mm}^{3}$, Plt $=7 \times 1000 / \mathrm{mm}^{3}$ ). Four days later, and in 
fetal color-doppler echocardiography, a 24-week female fetus was seen. Her major cardiac structures were normal, and the only finding was fetal tachycardia (175/min) that raised gynecologist concerns about fetal distress and maternal etiologies of the fetal tachycardia. She underwent bone marrow aspiration and biopsy (BMA/BX), which revealed non-M3 acute myeloid leukemia (AML)(Figure 1). After After considering a new case of AML, she was advised to think about the termination of pregnancy. Despite being aware of the risks that threatened her life and even that of the fetus, she did not agree with terminating the pregnancy or even starting the chemotherapy. During her admission, she encountered several problems such as perianal abscess and several cellulitis as well as gestational diabetes mellitus, which was eventually managed by insulin administration. After getting some antibiotics for her abscess and receiving excessive amounts of packed cell and platelet, she was discharged with her consent without getting any chemotherapy. Knowing that she did not follow the basic principles of personal protection and her attendance in high-risk places, she returned to our hospital about three weeks later with severe respiratory distress. Her pancytopenia got worsened. She received large amounts of blood product. Her LDH was elevated, and her lung CT-Scan showed findings compatible with Covid-19 infection (Figure 2). Her serologic tests (IgG and IgM antibodies against covid-19) were negative, which may be as a result of leukemia, but Her RT-PCR on the nasopharyngeal swab sample was positive with high viral load. While still insisting on not receiving chemotherapy, multi-drug treatment for Covid-19 infection was started for her using infliximab, Beta-Interferon, Hydrocortisone, and sofosbuvir. She was intubated the day before delivery because of her severe respiratory distress and abnormal arterial blood gas (ABG) results. After delivery, chemotherapy was started with Adriamycin and Cytosar. Two days after delivery, she expired.

On July 6, 2020 and following a hypertension crisis in the mother, an emergent cesarean section was performed. After general anesthesia, she underwent cesarean section, with unruptured amniotic fluid membrane, and while the isolation protocols were fully implemented, a girl was born at 31 weeks of gestational age. Her birth weight was $1,440 \mathrm{~g}$, and her head circumference was $27.5 \mathrm{~cm}$, both appropriate for gestational age, between 10th and 50th percentile on Fenton growth curve. Her first minute Apgar score was 1 , after resuscitation steps according to the current neonatal resuscitation program, her Apgar score reached 7 in the fifth minute of her life. After receiving continuous positive airway pressure, her Apgar reached 8 in the tenth minute of her life. Delayed cord clamping and skin to skin contact was not performed. Within minutes of placental expulsion, a sample of placental tissue from the fetal side was taken immediately after delivery, using an aseptic technique and sterile equipment.
The sample was transferred to the laboratory in viral transport media. The neonate was transferred to the NICU ward in an isolated room with negative pressure. All personal protection guidelines were performed for the nursing system and treatment staff. Cord blood gas analysis was normal. Because of mild to moderate subcostal retraction and tachypnea she needed supplementary oxygen with hood and blender using inspired oxygen fraction titrated gradually up to $30 \%$.

The first nasopharyngeal swab sample was collected after the baby was cleaned at the first 24 hours of her life. The second nasopharyngeal swab sample was collected on the third day of life. RT-PCR on both nasopharyngeal swab samples was positive with a high viral load similar to her mother (Figure 3 and Table 1). Nasopharyngeal swab and placental samples were examined for coronavirus RT-PCR. The swabs and tissue samples were placed in viral transport medium or sterile saline and stored at $4-8^{\circ} \mathrm{C}$ until they were sent to the laboratory to be processed within 4-12 hours of collection. RNA was extracted from the cell culture supernatants, and one-step TaqMan RT-PCR was performed in duplicate.

Her first CRP, CBC, and other laboratory data were all normal with no lymphopenia. She had some borderline blood sugar tests that were corrected by receiving dextrose water serum solution. She had elevated LDH levels up to $932 \mathrm{U} / \mathrm{ml}$, SGOT, and SGPT were in the normal range. Her brain, abdominopelvic sonography, and echocardiography were normal. On the second day of her life, repetitive episodes of apnea had occurred. Because of mild hypotonia, she underwent a lumbar puncture, which showed a normal pattern. Cerebrospinal fluid (CSF) was negative for SARS-CoV2, bacteria, fungi, and HSV 1 and 2. Her sepsis work-up did not reveal any abnormal results, and her blood, CSF, and urine culture were negative. Her serologic findings (IgG and IgM antibodies against covid-19) were negative on the fifth day of life. Serologic tests were repeated two weeks later, which showed negative results. During the admission, her need for supplementary oxygen was gradually tapered and episodes of feeding intolerance, which were defined as necrotizing enterocolitis (NEC), were managed uneventfully using traditional antibiotics without any special treatment for Covid-19. She only received azithromycin because of her prolonged need for supplementary oxygen and conjunctivitis. Her weight gain and head circumference growth pattern were both normal, and her prematurity care follow up, including retinopathy of prematurity (ROP) examination, were in progress (Figure 4).

\subsection{Special Neonatal Aspects}

In addition to routine prematurity care, her vaccination is also important. Moreover, her mother received 

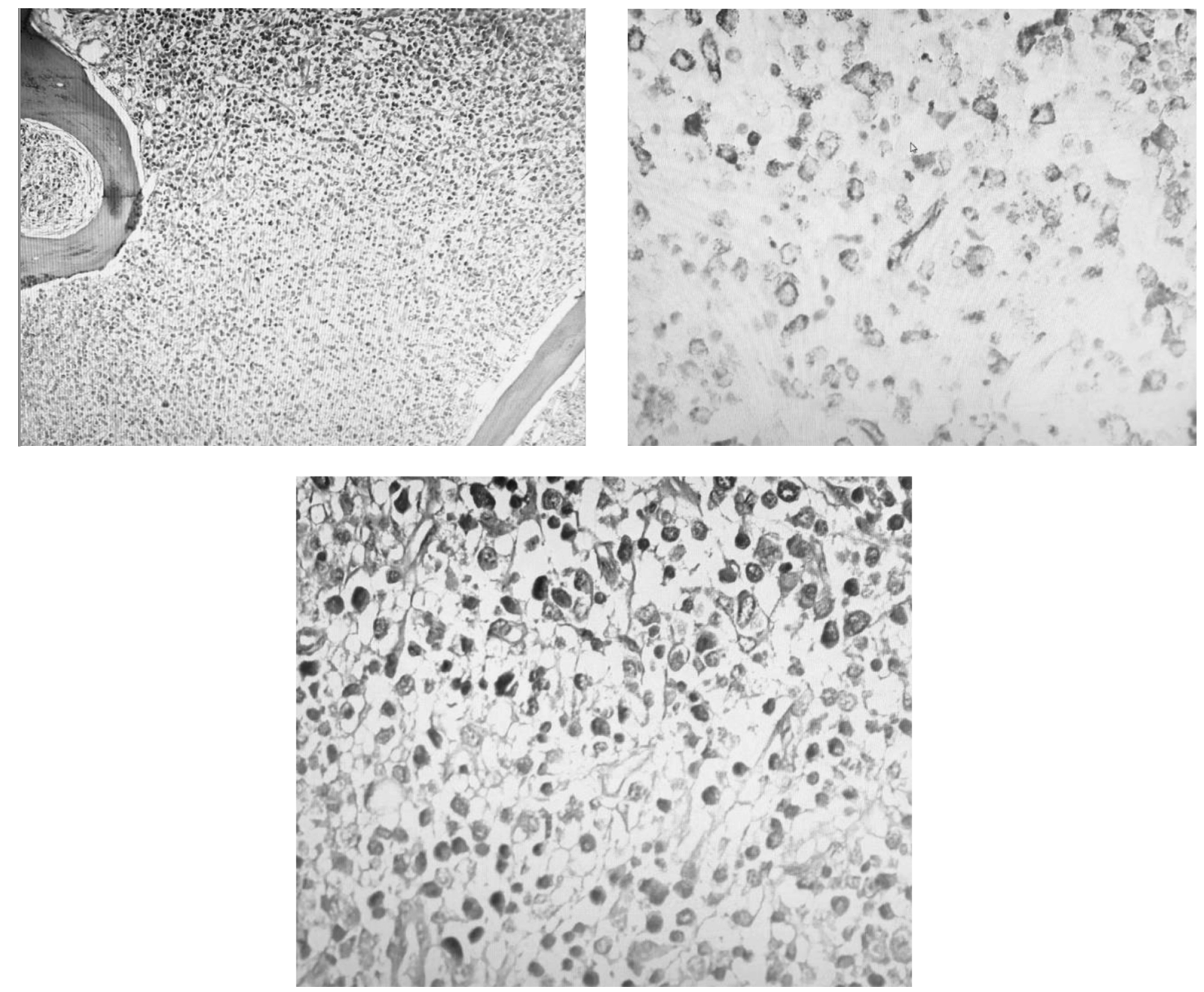

Figure 1. Some blasts are seen in bone marrow aspiration smear and bone marrow tissue with $100 \%$ cellularity, occupied sheets of mononuclear young cells with fewer recognizable myeloid cells and plasma cells. In IHC stains, over 50\% of marrow cells are positive for CD34, CD 117, and MPO.

infliximab for Covid-19 treatment. Infliximab is an antiTNF- $\alpha$ monoclonal antibody it is mostly used for the treatment of autoimmune diseases, and recently also as an immunomodulatory treatment of Covid-19. Its clearance half-life is 9.5 days, and it has placental transfer in the third trimester. The BCG vaccine contains a live attenuated form of Mycobacterium bovis. Various studies demonstrate that neonates whose mothers are being treated with infliximab or other biologic therapies, especially in the last trimester of pregnancy may have problems showing normal immunologic response to attenuated live vaccines. It is recommended to postpone all live vaccines until the infant is at least seven months old (9).

\section{Discussion}

There are not many reports on placental Covid-19 involvement up to now.

In March 2020, researchers in Paris reported a male neonate at 35-week of gestational age, born to a mother with Covid-19. RT-PCR of the placenta was positive. Mother was discharged in good condition six days after delivery, but suddenly after three days, his symptoms began with agitation, opisthotonus, increased muscular tone, and poor feeding. Like our case report, CSF was negative for SARS-CoV-2. Symptoms improved over three days. The samples obtained from the placenta showed higher viral load than those of amniotic fluid or blood samples obtained from the mother or her neonate. Ultimately, nasopharyngeal samples from the neonate showed higher viral load in 


\begin{tabular}{|c|c|c|c|}
\hline Sample Data & PCR Results & \multicolumn{2}{|c|}{ IgG and IgM Antibodies Against Covid-19 } \\
\hline \multicolumn{4}{|l|}{ Mother } \\
\hline \multirow[t]{2}{*}{ Nasopharyngeal } & Positive & $\operatorname{IgG}$ & Negative \\
\hline & & $\operatorname{IgM}$ & Negative \\
\hline \multicolumn{4}{|l|}{ Neonate } \\
\hline Nasopharyngeal $^{\text {a }}$ & Positive & $\operatorname{IgM}^{\mathrm{e}}$ & Negative \\
\hline Nasopharyngeal $^{\mathrm{b}}$ & Positive & $\operatorname{IgG}^{e}$ & Negative \\
\hline Nasopharyngeal $^{\mathrm{c}}$ & Negative & $\operatorname{IgM}^{\mathrm{f}}$ & Negative \\
\hline Nasopharyngeal $^{\mathrm{d}}$ & Negative & $\operatorname{IgG}{ }^{f}$ & Negative \\
\hline CSF & Negative & & \\
\hline Placenta & Positive & & \\
\hline \multicolumn{4}{|c|}{$\begin{array}{l}{ }^{a} \text { Nasopharyngeal refers to neonatal swab sample on the first day of her life. } \\
\text { b Nasopharyngeal refers to neonatal swab sample on the third day of her life. } \\
{ }^{c} \text { Nasopharyngeal refers to neonatal swab sample in the third week of her life. } \\
{ }^{d} \text { Nasopharyngeal refers to neonatal swab sample in the fourth week of her life. } \\
\text { e IgM and IgG refer to neonatal serologic findings on the fifth day of her life. } \\
\text { f IgM and IgG refer to neonatal serologic findings after two weeks. }\end{array}$} \\
\hline
\end{tabular}

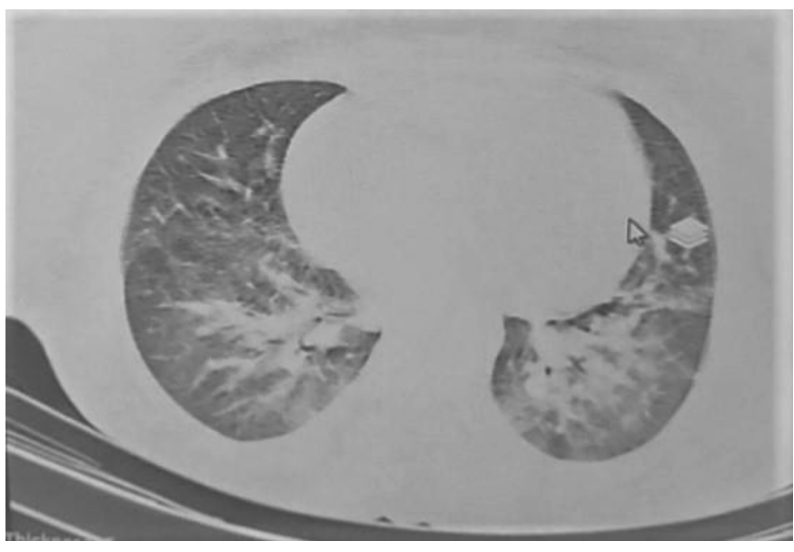

Figure 2. Maternal lung CT-Scan with multiple peripheral and severe periboronchovascular ground glass opacities in both lungs are seen, which is accompanied by hypervascularity and vascular dilatation in both sides in central and lower lobes, which could be in favor of viral pneumonia, including covid-19 infection.

RT-PCR curves on the 3rd and 18th day of life than that of the initial day of her life. Of note, during this period of time, the neonate was kept in full isolation condition in a negative pressure room (5). This case, just like our case report, was also one of the first cases of placental involvement, which can be used to prove vertical transmission.

There is another case report in which placental tissue PCR was positive for Covid-19, but by definition, it should be considered probable congenital infection hence the cord had not been tested. Mother had familial neutropenia, with a history of recurrent and repeated bacterial infection. She also had gestational diabetes mellitus. All of her infections were resolved with antibiotic treatment. The patient had fever, myalgia, inappetence, weariness, and dry cough. Her nasopharyngeal swab test with RT-PCR was positive for SARS-CoV-2. Placental swabs (both maternal and fetal sides) were also positive. Nasopharyngeal swabs, which were obtained from the neonate and after washing the neonate thoroughly, were positive for SARS-CoV-2 on the day of birth, day 2, and day 7. Before taking the samples, the neonate did not have any contact with his mother. The neonate was neutropenic and had mild hypothermia, intermittent hypoglycemia, and episodes of feeding intolerance, just like our case. Routine antibiotic treatment started empirically. This case, just like ours, represented a case of probable congenital SARS-CoV-2 infection in a newborn baby (7).

A miscarriage case was evaluated at Lausanne University Hospital. She was a 28-year-old woman at 19 weeks of her gestation. Her symptoms were fever, myalgia, weariness, dysphagia, diarrhea, and dry cough. A nasopharyngeal swab sample showed a positive result for SARS-CoV2. Two days later, her uterine contractions started, her fever was resistant, and there was no amelioration seen in her condition. A stillborn infant was delivered vaginally. Placenta was positive for SARS-CoV-2. Other samples, including the mother's blood, urine, and vaginal discharge, were negative for SARS-CoV-2, except for the nasopharyngeal swab, which was positive. Having negative results for other swab tests, it is improbable to attribute the placental tissue positive result in delivery time or sampling contamination for SARS-CoV-2. No other reason was found for fetal death (10). In another study among 32 pregnant women, three placental samples had positive PCR results. All three 


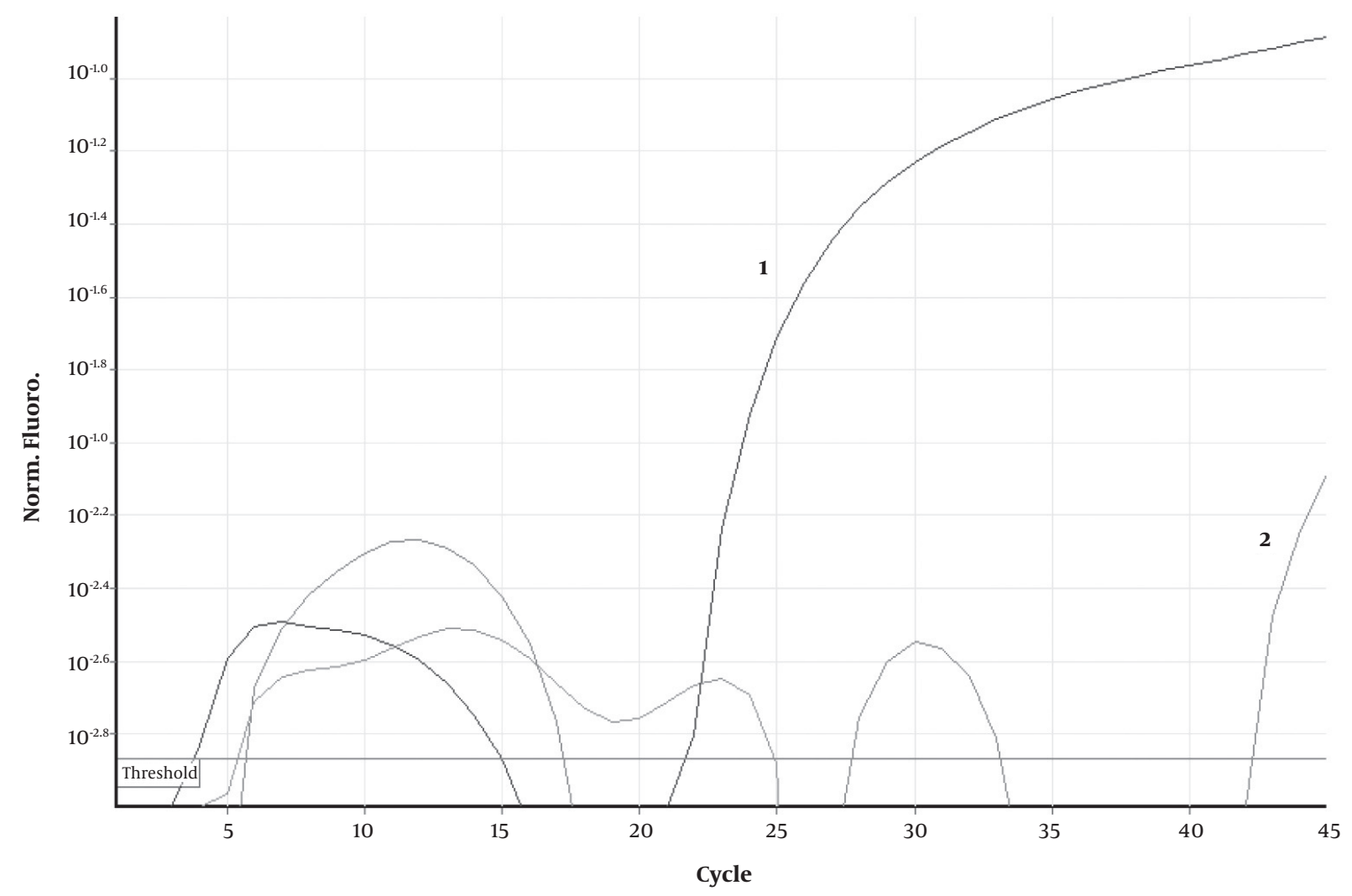

Figure 3. Real-time polymerase chain reaction results related to the fetal side of placental tissue. The curve marked as 2 is related to fetal side of placenta, as shown this curve is above the threshold, indicated by a continuous straight red line, which its pattern is similar to the positive control curve marked as 1 , which is a SARS-CoV-2 culture supernatant.

positive samples were obtained from mothers who had severe Covid-19 during delivery. In women with severe to critical COVID-19 at the time of delivery, just like our case report. The neonates showed no clinical signs of vertical transmission (11). If the contact happened during delivery, more time would be needed for the virus to go through an incubation period before tests demonstrated positive results. Hence, nasopharyngeal tests soon after birth may not be an optimal way to assess the vertical route of transmission. So it may take longer for tests to disclose positive $\operatorname{PCR}(11,12)$.

There are some other ways to prove that SARS-CoV-2 may be transmitted vertically if IgM antibodies were found in blood drawn from neonates early after birth (1). It is important to remind that antibody tests have not been thoroughly assessed for sensitivity and specificity $(8,13)$. There are also other studies that have addressed the issue of vertical transmission of Covid-19, but in most of them, no evidence was found for intrauterine transmission of the virus, and the majority of neonates born to mothers who were infected with SARS-CoV-2 were negative according to PCR test

\section{$(9,14-17)$.}

\subsection{Limitations}

Timely and clean sampling from not contaminated amniotic fluid and cord blood is an important challenge. An accurate transfer process in appropriate media from sampling site to the laboratory is crucial. In this study, we did not collect cord blood, amniotic fluid, and amniotic membrane samples, which are critical to proving vertical transmission definitely.

\section{Footnotes}

Authors' Contribution: Choobdar F.A and Ghassemzadeh.M conceived and designed the evaluation and drafted the manuscript. Abbariki.E and Attarian.M hepld for collecting data. Ghanbari.B and Azarbin.Z gave us accessibility to data and helped to draft the manuscript. Nateghian.A re-evaluated the clinical data, revised the manuscript. Shah Hamzehi S. collected the Para clinical data, and helped us reporting radiologic findings. 


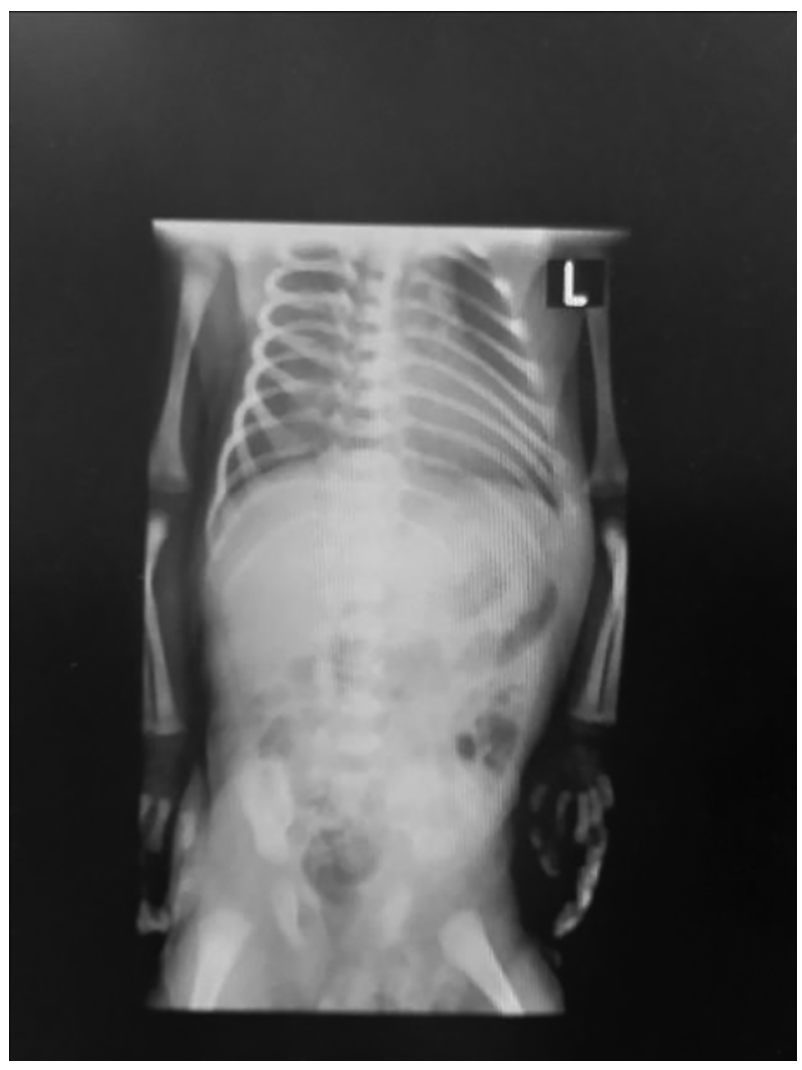

Figure 4. Chest X-Ray of the neonate in the first day of her life, relatively hyperinflation of both lungs without obvious infiltration is present.

Hashemi R.M collected pathologic data and PCR curves and their analysis.

Conflict of Interests: There is no conflict of interest in this manuscript

Ethical Approval: Written informed consent was obtained from the father for the publication of this report and performing laboratory tests on the neonate.

Funding/Support: We did not get any financial support for this case report.

\section{References}

1. Dong L, Tian J, He S, Zhu C, Wang J, Liu C, et al. Possible vertical transmission of SARS-CoV-2 from an infected mother to her newborn. JAMA. 2020;323(18):1846-8. doi: 10.1001/jama.2020.4621. [PubMed: 32215581]. [PubMed Central: PMC7099527].

2. Wang W, Xu Y, Gao R, Lu R, Han K, Wu G, et al. Detection of SARS-CoV2 in different types of clinical specimens. JAMA. 2020;323(18):1843-4. doi: 10.1001/jama.2020.3786. [PubMed: 32159775]. [PubMed Central: PMC7066521].

3. Woo PC, Lau SK, Wong BH, Tsoi HW, Fung AM, Chan KH, et al. Detection of specific antibodies to severe acute respiratory syndrome
(SARS) coronavirus nucleocapsid protein for serodiagnosis of SARS coronavirus pneumonia. I Clin Microbiol. 2004;42(5):2306-9. doi: 10.1128/jcm.42.5.2306-2309.2004. [PubMed: 15131220]. [PubMed Central: PMC404667].

4. Xu W, Li J, He X, Zhang C, Mei S, Li C, et al. The diagnostic value of joint detection of serum IgM and IgG antibodies to 2019-nCoV in 2019-nCoV infection. Chinese J Lab Med. 2020;43(3).

5. Vivanti AJ, Vauloup-Fellous C, Prevot S, Zupan V, Suffee C, Do Cao J, et al. Transplacental transmission of SARS-CoV-2 infection. Nat Commun. 2020;11(1):3572. doi: 10.1038/s41467-020-17436-6. [PubMed: 32665677]. [PubMed Central: PMC7360599].

6. Shah PS, Diambomba Y, Acharya G, Morris SK, Bitnun A. Classification system and case definition for SARS-CoV-2 infection in pregnant women, fetuses, and neonates. Acta Obstet Gynecol Scand 2020;99(5):565-8. doi: 10.1111/aogs.13870. [PubMed: 32277845]. [PubMed Central: PMC7262318].

7. Kirtsman M, Diambomba Y, Poutanen SM, Malinowski AK, Vlachodimitropoulou E, Parks WT, et al. Probable congenital SARS-CoV-2 infection in a neonate born to a woman with active SARS-CoV-2 infection. CMAJ. 2020;192(24):E647-50. doi:10.1503/cmaj.200821. [PubMed: 32409520]

8. Wang C, Zhou YH, Yang HX, Poon LC. Intrauterine vertical transmission of SARS-CoV-2: what we know so far. Ultrasound Obstet Gynecol. 2020;55(6):724-5. doi: 10.1002/uog.22045. [PubMed: 32266753]. [PubMed Central: PMC7262402].

9. Cheent K, Nolan J, Shariq S, Kiho L, Pal A, Arnold J. Case Report: Fatal case of disseminated BCG infection in an infant born to a mother taking infliximab for Crohn's disease. J Crohns Colitis. 2010;4(5):603-5. doi: 10.1016/j.crohns.2010.05.001. [PubMed: 21122568].

10. Baud D, Greub G, Favre G, Gengler C, Jaton K, Dubruc E, et al. Second-trimester miscarriage in a pregnant woman with SARS-CoV2 infection. JAMA. 2020;323(21):2198-200. doi: 10.1001/jama.2020.7233. [PubMed: 32352491]. [PubMed Central: PMC7193526].

11. Penfield CA, Brubaker SG, Limaye MA, Lighter J, Ratner AJ, Thomas $\mathrm{KM}$, et al. Detection of severe acute respiratory syndrome coronavirus 2 in placental and fetal membrane samples. Am J Obstet Gynecol MFM. 2020;2(3):100133. doi: 10.1016/j.ajogmf.2020.100133. [PubMed: 32391518]. [PubMed Central: PMC7205635].

12. Qiu L, Liu X, Xiao M, Xie J, Cao W, Liu Z, et al. SARS-CoV-2 is not detectable in the vaginal fluid of women with severe COVID-19 infection. Clin Infect Dis. 2020;71(15):813-7. doi: 10.1093/cid/ciaa375. [PubMed: 32241022]. [PubMed Central: PMC7184332].

13. Kimberlin DW, Stagno S. Can SARS-CoV-2 infection be acquired in Utero?: More definitive evidence is needed. JAMA. 2020;323(18):17889. doi: 10.1001/jama.2020.4868. [PubMed: 32215579].

14. Karimi-Zarchi M, Neamatzadeh H, Dastgheib SA, Abbasi H, Mirjalili SR, Behforouz A, et al. Vertical transmission of coronavirus disease 19 (COVID-19) from infected pregnant mothers to neonates: A review. Fetal Pediatr Pathol. 2020;39(3):246-50. doi: 10.1080/15513815.2020.1747120. [PubMed:32238084]. [PubMed Central: PMC7157948].

15. Masmejan S, Pomar L, Favre G, Panchaud A, Giannoni E, Greub G, et al. Vertical transmission and materno-fetal outcomes in 13 patients with coronavirus disease 2019. Clin Microbiol Infect. 2020;26(11):1585-7. doi: 10.1016/j.cmi.2020.06.035. [PubMed:32652239]. [PubMed Central: PMC7341030].

16. Alzamora MC, Paredes T, Caceres D, Webb CM, Valdez LM, La Rosa M. Severe COVID-19 during pregnancy and possible vertical transmission. Am J Perinatol. 2020;37(8):861-5. doi: 10.1055/s-0040-1710050. [PubMed: 32305046]. [PubMed Central: PMC7356080].

17. Abolhasan Choobdar F, Ghassemzadeh M, Abbariki E, Attarian M. Clinical characteristics and report of seven Iranian neonates born to mothers with COVID-19. Am J Pediatr. 2020;6(3):303. doi: 10.11648/j.ajp.20200603.32. 